1 A sex difference in the context-sensitivity of dominance perceptions

2

3 CHRISTOPHER D WATKINS ${ }^{1}$, LISA M DEBRUINE ${ }^{2}$, DAVID R FEINBERG $^{3}$ \&

4 BENEDICT C JONES ${ }^{2}$

5

$6{ }^{1}$ Division of Psychology, School of Social and Health Sciences, University of

7 Abertay, Scotland, UK.

8

$9 \quad{ }^{2}$ Face Research Laboratory, Institute of Neuroscience and Psychology,

10 University of Glasgow, 58 Hillhead Street, Glasgow, G12 8QB.

11

$12{ }^{3}$ Department of Psychology, Neuroscience and Behaviour, McMaster

13 University, Hamilton, Ontario, L8S4L8, Canada.

14

15

16

17 Corresponding author

18 Christopher Watkins, Division of Psychology, School of Social and Health

19 Sciences, University of Abertay, Scotland, UK

20 Email: c.watkins@abertay.ac.uk

21

22 Full Citation: Watkins, C.D., DeBruine, L.M., Feinberg, D.R. and Jones, B.C.

23 (2013). A sex difference in the context-sensitivity of dominance perceptions.

24 Evolution and Human Behavior, 34(5): 366-372. 


\section{A sex difference in the context-sensitivity of dominance perceptions}

\section{(1)}

\section{Abstract}

Although dominance perceptions are thought to be important for effective social interaction, their primary function is unclear. One possibility is that they simply function to identify individuals who are capable of inflicting substantial physical harm, so that the perceiver can respond to them in ways that maximize their own physical safety. Another possibility is that they are more specialized, functioning primarily to facilitate effective direct (i.e., violent) intrasexual competition for mates, particularly among men. Here we used a priming paradigm to investigate these two possibilities. Facial cues of dominance were more salient to women after they had been primed with images of angry men, a manipulation known to activate particularly strong self-protection motivations, than after they had been primed with images of angry women or smiling individuals of either sex. By contrast, dominance cues were more salient to men after they had been primed with images of women than when they had been primed with images of men (regardless of the emotional expressions displayed), a manipulation previously shown to alter men's impressions of the sex ratio of the local population. Thus, men's dominance perceptions appear to be specialized for effective direct competition for mates, while women's dominance perceptions may function to maximize their physical safety more generally. Together, our results suggest that men's and women's dominance perceptions show different patterns of context-sensitivity and, potentially, shed new light on the routes through which violence and intrasexual competition have shaped dominance perceptions. 


\section{2}

53

\section{Introduction}

Dominance perceptions are fundamental to human social interaction (e.g.,

Oosterhof \& Todorov, 2008; Puts, 2010). However, although previous research suggests that people from different cultures (e.g., Keating et al., 1981; Perrett et al., 1998; Undurraga et al., 2010) and people of diverse ages (e.g., Keating \& Bai, 1986) judge others' dominance in similar ways, the specific function of dominance perceptions is still poorly understood. Some researchers have suggested that dominance perceptions simply function to identify individuals who are capable of inflicting substantial physical harm, so that the perceiver can respond to them in ways that maximize their own physical safety (e.g., by avoiding them, Oosterhof \& Todorov, 2008). Alternatively, dominance perceptions may be more specialized, functioning primarily to facilitate effective direct (i.e., violent) intrasexual competition for mates, particularly among men (Puts, 2010). Because distinguishing between these two proposals could provide important insight into the routes through which physical violence and intrasexual competition for mates have shaped the visuo-cognitive processes that support social interactions, the current research tested these two suggestions about the primary function of dominance perceptions.

Self-protection motivations are hypothesized to moderate aspects of social cognition and perception that have implications for survival (e.g., Kenrick et al., 2010). For example, people are particularly quick to classify angry expressions in face images, especially when the angry expressions are presented in the context of male faces (Becker et al., 2007). These findings 
suggest that viewing images of angry faces, and of angry men in particular, activates self-protection motivations (Kenrick et al., 2010; see also Ackerman et al., 2006). If dominance perceptions function primarily to identify individuals capable of inflicting physical harm, as some researchers have suggested (e.g., Oosterhof \& Todorov, 2008), then activating self-protection motivations should increase the salience of dominance cues. Thus, priming participants with angry male faces should increase the extent to which participants ascribe dominance to individuals displaying cues associated with physical dominance more than would priming participants with images of angry female faces or smiling faces of either sex. Additionally, this effect of priming participants with angry male, but not angry female, faces could be sex-specific in other ways. For example, activating self-protection motivations may have greater effects on the cognitions and perceptions of individuals who are less well equipped (or perceive themselves to be less well equipped) to defend themselves physically (e.g., Fox et al., 2001; Kenrick et al., 2010). Given sex differences in both physical strength and aggression (see, e.g., Archer, 2009; Sell et al., 2009), activating self-protection motivations may have a greater effect on women's perceptions of others' dominance than it will on men's perceptions of others' dominance.

\section{While testing the effect of activating self-protection motivations on the} salience of dominance cues would test for evidence that dominance perceptions simply function to identify individuals capable of inflicting physical harm, other types of primes could be used to test the proposal that dominance perceptions serve a more specialized purpose and function primarily to 
102 minimize the potential costs of direct intrasexual competition for mates,

103 particularly among men (see, e.g., Puts, 2010). Although competition among

104 men tends to be increased in societies with a greater proportion of men than

105 women (i.e., societies with male-biased sex ratios), this competition is

106 generally indirect (i.e., non-violent) and focused on gaining access to

107 economic resources (e.g., Barber, 2009; Del Giudice, 2012). Indeed,

108 Griskevicius et al. (2012) recently showed that priming men with cues to a

109 male-biased sex ratio increased the extent to which men were willing to

110 sacrifice larger financial gains in the future for smaller, immediate gains (i.e.,

111 the extent to which they seek immediate access to economic resources). By

112 contrast, in societies with female-biased sex ratios, relationship commitment

113 tends to be relatively low and sexual promiscuity relatively common (Barber,

$1142000,2009,2011$; Schmitt, 2005), which increases direct (i.e., violent)

115 competition for mates among men, at least in modern societies (Barber, 2011;

116 Del Giudice, 2012). Indeed, this may explain why rates of violent crime tend to

117 be higher in countries with more female-biased sex ratios (Barber, 2000,

$1182009,2011)$.

120 Several recent studies have shown that watching slideshows consisting

121 primarily of either images of men or images of women alters behavioral

122 responses, such as attractiveness judgments or financial decisions, in ways

123 that suggest participants use their recent visual experience to gauge the sex

124 ratio of the local population (Griskevicius et al., 2012; Watkins et al., 2012a).

125 These findings demonstrate that priming paradigms can be used to explore

126 the effects of cues to the sex ratio of the local population on aspects of social 
127 behavior and perception (Griskevicius et al., 2012; Watkins et al., 2012a).

128 Thus, if dominance perceptions primarily function to minimize the potential 129 costs of direct competition for mates among men (e.g., Puts, 2010), cues of

130 others' dominance may be more salient to men in environments with a

131 female-biased sex ratio (i.e., after they have been primed with a slideshow of

132 images of women's faces) than in environments with a male-biased sex ratio

133 (i.e., after they have been primed with a slideshow of images of men's faces).

134 This effect could be specific to judgments of men's dominance or could occur

135 for judgments of others' dominance more generally. For example, while some

136 aspects of men's facultative responses to facial cues of dominance appear to

137 be specific to judgments of other men's dominance (Watkins et al., 2010a),

138 other studies suggest that men are also sensitive to cues of dominance of

139 women (e.g., Perrett et al., 1998; Sell et al., 2009).

141 While the prediction that cues of others' dominance will be more salient to

142 men in environments with a female-biased sex ratio may initially seem to be

143 somewhat at odds with Griskevicius et al's (2012) finding that priming men

144 with cues to a male-biased sex ratio increased the extent to which men

145 favored smaller, immediate gains over larger gains in the future, Griskevicius

146 et al's (2012) finding presumably reflects the well-established correlation

147 between male-biased sex ratios and indirect (i.e., non-violent) competition

148 (Barber, 2009; Del Giudice, 2012). By contrast, our prediction that priming

149 men with cues that there is a greater proportion of women than men in the

150 local population will increase the extent to which dominance cues are salient

151 is based on the reported positive correlations between female-biased sex 
152 ratios and measures of the intensity of direct (i.e., violent) competition

153 (Barber, 2000, 2009, 2011).

154

155 To test the predictions described above, we investigated the effects of priming

156 with images of angry men, smiling men, angry women, or smiling women on

157 men's and women's perceptions of others' dominance. So that we could 158 assess the effects of these different types of primes on the salience of cues of 159 physical dominance (i.e., the extent to which participants perceived physically 160 dominant individuals to be more dominant than less physically dominant 161 individuals, Watkins \& Jones, 2012), we assessed participants' perceptions of

162 the dominance of masculinized versus feminized versions of men's and 163 women's faces. We chose this image manipulation (masculinized versus 164 feminized) because many recent studies have demonstrated that masculine 165 characteristics are positively correlated with measures of actual physical 166 dominance, such as strength and aggression (e.g., Fink et al., 2007;

167 Windhager et al., 2011; Puts et al., 2011), and because masculinized versions 168 of faces are reliably perceived to be more dominant than feminized versions 169 (Jones et al., 2010; Perrett et al., 1998; Watkins et al., 2010a).

171 Methods

172 Participants

173 One hundred women (mean age $=20.95$ years, $S D=3.13$ years) and 100 men 174 (mean age=22.49 years, SD=3.58 years) completed the experiment online.

175 Participants were recruited from links on social bookmarking websites, such 176 as www.stumbleupon.com. Previous research on perceptions of facial 
177 dominance has demonstrated that laboratory and online studies produce

178 equivalent results (Senior et al., 1999a, 1999b; see also Watkins et al., 2010a, 179 2010b).

\section{Face stimuli}

182 The methods we used to manufacture stimuli to assess perceptions of the 183 dominance of masculinized versus feminized versions of men's and women's 184 faces have been used in many previous studies of dominance perceptions 185 (e.g., DeBruine et al., 2006; Perrett et al., 1998; Watkins \& Jones, 2012). 186 Manipulating sexually dimorphic shape cues in face images using these 187 methods has been shown to alter perceptions of men's and women's facial 188 dominance in the predicted manner (e.g., DeBruine et al., 2006; Watkins et 189 al., 2010a, 2012b). Moreover, responses to masculinity stimuli manufactured 190 using these methods are very similar to responses to facial masculinity stimuli 191 that were manufactured using other methods (e.g., DeBruine et al., 2006, 192 2010).

194 First, we manufactured a male prototype (i.e., average) face by using 195 specialist software (Tiddeman et al., 2001) to average the shape, color, and 196 texture information from images of 50 young white men's faces. A female 197 prototype face was also manufactured in this way by averaging the shape, 198 color, and texture information from images of 50 young white women's faces. 199 The 100 individual face photographs (50 male and 50 female) were taken 200 under standardized lighting conditions and against a constant background. 
201 Individuals posed for these photographs with neutral expressions and direct 202 gaze.

204 Next, we randomly selected 10 male and 10 female images from the set of 205100 face images. We created a masculinized and a feminized version of each 206 of the 10 individual male and 10 individual female images by adding or 207 subtracting $50 \%$ of the linear (i.e., vector) differences in 2D shape between 208 symmetrized versions of the male and female prototypes to (or from) each 209 individual image. This process created 20 pairs of face images in total (10 210 male pairs and 10 female pairs), with each pair consisting of a masculinized

211 and a feminized version of one of the individual face images. Examples of 212 these stimuli are shown in Figure 1. Note that our masculinized and feminized 213 versions of faces differed in sexually dimorphic shape characteristics only 214 (i.e., were matched in other regards, such as identity, color, texture, Tiddeman 215 et al., 2001).

INSERT FIGURE 1 AROUND HERE

\section{Manipulation check}

220 We conducted an initial pilot study to check that the masculinized and

221 feminized versions of faces differed reliably in perceived masculinity. In this 222 pilot study, the 20 pairs of face images (each pair consisting of a masculinized 223 and feminized version of the same face) were presented to 52 women and 21 224 men (mean age $=24.55$ years, $S D=8.73$ years), who were instructed to 225 indicate which face in each pair looked more masculine. Pairs of faces were 
226 presented in a fully randomized order and the side of the screen on which a

227 given image was shown was also randomized. One-sample t-tests were used

228 to compare the proportion of trials on which participants correctly identified the

229 masculinized face with what would be expected by chance alone (i.e., 0.5).

230 These analyses confirmed that the masculinized versions of faces were

231 perceived to be more masculine than feminized versions of faces when

232 judging men's $(\mathrm{t}(72)=23.13, \mathrm{p}<.001, \mathrm{~d}=2.71, \mathrm{M}=.90, \mathrm{SEM}=.02)$ and women's

$233(\mathrm{t}(72)=24.72, \mathrm{p}<.001, \mathrm{~d}=2.89, \mathrm{M}=.91, \mathrm{SEM}=.01)$ masculinity. Corresponding

234 by-items analyses, in which face pairs, rather than participants, served as the

235 primary unit of analysis, showed the same pattern of results (men's faces:

$236 \mathrm{t}(9)=24.79, \mathrm{p}<.001, \mathrm{~d}=7.77, \mathrm{M}=.90, \mathrm{SEM}=.02$; women's faces: $\mathrm{t}(9)=32.11$,

$237 \mathrm{p}<.001, \mathrm{~d}=10.20, \mathrm{M}=.91, \mathrm{SEM}=.01)$. These results are consistent with prior

238 work showing that manipulating sexually dimorphic shape cues in face images

239 using these methods alters perceptions of men's and women's facial

240 masculinity (e.g., DeBruine et al., 2006; Welling et al., 2007, 2008).

242 A second pilot study was also conducted, in which 125 participants (64

243 women and 61 men, mean age $=21.96$ years, $S D=3.08$ years) were instructed

244 to indicate which face in each pair looked more dominant, rather than

245 masculine. By-subjects analyses confirmed that the masculinized versions of

246 faces were perceived to be more dominant than feminized versions of faces

247 when judging men's $(\mathrm{t}(124)=17.93, \mathrm{p}<.001, \mathrm{~d}=1.60, \mathrm{M}=.81, \mathrm{SEM}=.02)$ and

248 women's $(\mathrm{t}(124)=3.69, \mathrm{p}<.001, \mathrm{~d}=0.33, \mathrm{M}=.60, \mathrm{SEM}=.03)$ dominance.

249 Corresponding by-items analyses also showed this pattern of results (men's

250 faces: $t(9)=17.21, p<.001, d=5.42, M=.81$, SEM=.02; women's faces: 
$251 \mathrm{t}(9)=8.02, \mathrm{p}<.001, \mathrm{~d}=2.50, \mathrm{M}=.60, \mathrm{SEM}=.01)$. These results are consistent

252 with prior work showing that masculinizing shape cues in face images using

253 these methods alters perceptions of men's and women's dominance (e.g.,

254 DeBruine et al., 2006; Jones et al., 2010; Perrett et al., 1998; Watkins et al., 255 2010a).

256

\section{Procedure}

258 The main experiment consisted of three parts; an initial pre-priming

259 dominance perception test, a priming phase in which participants watched a

260 slideshow of male or female face images displaying either angry or smiling

261 expressions, and a post-priming dominance perception test.

263 In the pre-priming dominance perception test, each of the 200 participants

264 were shown the 20 pairs of face images (10 male pairs and 10 female pairs,

265 each pair consisting of a masculinized and feminized version of the same

266 face) and were instructed to indicate which face in each pair looked more

267 dominant. Trial order and the side of the screen on which any given image

268 was presented were fully randomized. The purpose of this pre-priming test

269 was to obtain a baseline estimate of participants' dominance perceptions, so

270 that we could control for the possible effects of pre-existing individual

271 differences in dominance judgments (e.g., Watkins et al., 2010b, 2012b).

273 Immediately after completing the pre-priming test, each participant watched a

274 slideshow of images depicting either 30 angry male faces, 30 angry female

275 faces, 30 smiling male faces, or 30 smiling female faces. These angry and 
276 smiling faces were obtained from the Karolinska Directed Emotional Faces

277 (KDEF) image set (Lundqvist \& Litton, 1998). In the slideshows, each of the

27830 faces shown was presented onscreen for 2 seconds (i.e., each slideshow

279 lasted 60 seconds in total) and the order in which the images were presented

280 was fully randomized. Following previous work that used similar slideshows to

281 manipulate cues to the nature of the local population (e.g., Jones et al., 2007;

282 Watkins et al., 2012a), participants were simply instructed to watch the

283 images closely. The 100 women and 100 men who took part in the

284 experiment were randomly allocated to one of the four slideshows. Previous

285 work has successfully shown images of faces displaying emotional

286 expressions to experimentally manipulate participants' motivations (e.g.,

287 Ackerman et al., 2006; Becker et al., 2007), while other work has successfully

288 shown images of either male or female faces in order to experimentally

289 manipulate cues to the sex ratio of the local population (Griskevicius et al.,

290 2012; Watkins et al., 2012a).

292 Immediately after viewing the slideshow (i.e., immediately after completing the

293 priming phase of the experiment), participants completed a post-priming

294 dominance perception test that was identical to the pre-priming test.

\section{Results}

297 For each participant, we calculated the proportion of trials on which they

298 chose masculinized faces as more dominant than feminized faces when

299 judging men's faces in the pre-priming test, women's faces in the pre-priming 
300 test, men's faces in the post-priming test, and women's faces in the post-

301 priming test. These scores are summarized in Table 1.

305 Consistent with prior work (e.g., Perrett et al., 1998; Watkins et al., 2010a), one-sample t-tests comparing the pre-priming test scores with what would be expected by chance alone (i.e., 0.5 ) showed that participants generally perceived masculinized faces to be more dominant than feminized faces at the start of the experiment when judging both men's faces $(t(199)=27.93$, $\mathrm{p}<.001, \mathrm{~d}=1.98, \mathrm{M}=.86, \mathrm{SEM}=.01)$ and women's faces $(\mathrm{t}(199)=2.81, \mathrm{p}=.005$, $\mathrm{d}=0.20, \mathrm{M}=.57, \mathrm{SEM}=.02$ ). Also consistent with prior work (e.g., Watkins et al., 2010a), this effect of facial masculinity on dominance perceptions in the pre-priming tests was significantly greater for judgments of men's dominance than women's dominance $(t(199)=13.56, p<.001, d=0.96)$. Repeating these analyses using Wilcoxon signed ranks tests in place of t-tests showed the same pattern of significant results.

318 Next, scores on the dominance perception test were analyzed using a mixed 319 design ANOVA with the within-subjects factors sex of face judged (male, 320 female) and test phase (pre-priming, post-priming) and the between-subjects 321 factors priming emotion (angry, smiling), priming sex (male, female), and 322 participant sex (male, female). This analysis revealed a significant main effect 323 of sex of face judged $\left(F(1,192)=172.89, p<.001\right.$, partial eta $\left.{ }^{2}=.47\right)$, which 324 reflected the general tendency to attribute dominance to masculinized faces 
325 more often when judging men's faces $(M=.86, S E M=.01)$ than when judging

326 women's faces $(M=.57, \mathrm{SEM}=.02)$. There was also a significant three-way

327 interaction among test phase, priming sex, and participant sex

$328\left(F(1,192)=6.89, p=.009\right.$, partial eta $\left.{ }^{2}=.04\right)$, which was qualified by the predicted

329 significant four-way interaction among test phase, priming emotion, priming

330 sex, and participant sex $\left(F(1,192)=5.79, p=.017\right.$, partial eta $\left.{ }^{2}=.03\right)$. No other

331 effects were significant or approached significance (all $F<1.30$, all $p>.25$, all

332 partial eta ${ }^{2}<.01$ ), except for a five-way interaction among test phase, sex of

333 face judged, priming emotion, priming sex, and participant sex that

334 approached significance $\left(F(1,192)=3.91, p=.050\right.$, partial eta $\left.{ }^{2}=.02\right)$. Since we

335 had no specific a priori prediction about the effects of sex of face judged, we

336 did not explore the possible five-way interaction further in our main analyses.

337 Indeed, Stevens (2007) recommends against exploring very high order

338 interactions unless they were a strong a priori prediction. We note here,

339 however, that repeating the ANOVAs we conducted to interpret the four-way

340 interaction among test phase, priming emotion, priming sex, and participant

341 sex with sex of face judged included as an additional within-subjects factor did

342 not alter our findings or reveal any effects of (or interactions involving) sex of

343 face judged (see additional analyses below). The five-way interaction

344 reflected the priming effect that was observed for male participants tending to

345 be greater for judgments of men's than women's faces (although not

346 significantly so).

348 To interpret the significant four-way interaction among test phase, priming

349 emotion, priming sex, and participant sex we conducted separate ANOVAs for 
350 male and female participants with the within-subjects factor test phase (pre-

351 priming, post-priming) and the between-subjects factors priming emotion

352 (angry, smiling) and priming sex (male, female). Scores on the dominance

353 perception tests were collapsed across the factor sex of face judged for these

354 analyses.

355

356 The analysis for female participants revealed a significant three-way

357 interaction among test phase, priming emotion, and priming sex

$358\left(F(1,96)=5.94, p=.017\right.$, partial eta $^{2}=.06$, Figure 2$)$ and no other significant

359 effects (all $\mathrm{F}<2.35$, all $\mathrm{p}>.13$, all partial eta $^{2}<.025$ ). For women allocated to the

360 angry priming emotion conditions, there was a significant interaction between

361 the effects of test phase and priming sex $(F(1,48)=6.77, p=.012$, partial

362 eta $\left.{ }^{2}=.12\right)$; women who were primed with angry male images $(\mathrm{t}(24)=2.30$,

$363 p=.030, d=0.46)$, but not those who were primed with angry female images

$364(t(24)=-1.68, p=.107, d=0.33)$, significantly increased the proportion of trials on

365 which they chose masculinized faces as more dominant between the pre-

366 priming and post-priming tests. For women allocated to the smiling priming

367 emotion conditions, there were no significant effects of test phase or priming

368 sex and the interaction between these variables was not significant (all

$369 \mathrm{~F}<0.50$, all $\mathrm{p}>.48$, all partial eta $\left.{ }^{2}<.010\right)$. Moreover, neither women who were

370 primed with smiling male images $(t(24)=-0.41, p=.69, d=0.08)$ nor women who

371 were primed with smiling female images $(t(24)=0.59, p=.56, d=0.12)$

372 significantly increased the proportion of trials on which they chose

373 masculinized faces as more dominant between the pre-priming and post-

374 priming tests. Together, these analyses show that the salience of facial cues 
375 of dominance was increased in women primed with angry male faces, but not

376 in women who were allocated to the other priming conditions. Repeating the

377 initial ANOVA for female participants with sex of face judged included as an

378 additional within-subjects factor did not alter the pattern of significant results

379 or reveal any interactions involving sex of face judged (all $F<1.15$, all $p>.28$,

380 all partial eta $\left.{ }^{2}<.013\right)$. Repeating the paired-samples analyses using Wilcoxon

381 signed ranks tests in place of t-tests showed the same pattern of significant

382 results.

383

INSERT FIGURE 2 AROUND HERE

385

386 The analysis for male participants revealed a significant two-way interaction

387 between test phase and priming sex $\left(F(1,96)=4.84, p=.030\right.$, partial eta ${ }^{2}=.05$,

388 Figure 3 ) and no other significant effects (all $F<0.90$, all $p>.34$, all partial

389 eta $\left.^{2}<.010\right)$. Men allocated to the female priming sex conditions tended to

390 increase the proportion of trials on which they chose masculinized faces as

391 the more dominant between the pre-priming and post-priming tests

$392(t(49)=1.53, p=.13, d=.22)$, while men allocated to the male priming sex

393 conditions tended to decrease the proportion of trials on which they chose

394 masculinized faces as the more dominant between the pre-priming and post-

395 priming tests $(\mathrm{t}(49)=-1.66, \mathrm{p}=.10, \mathrm{~d}=.24)$. Wilcoxon signed ranks tests also

396 showed this pattern of results. Note that, although neither of the individual

397 changes between the pre-priming and post-priming tests was actually

398 significant, these analyses of men's responses confirm that the effects of

399 priming men with images of women's or men's faces were significantly 
400 different from each other and occurred regardless of the emotional

401 expressions displayed by the priming images. Repeating the initial ANOVA for

402 male participants with sex of face judged included as an additional within-

403 subjects factor did not alter the pattern of significant results or reveal any

404 three- or four-way interactions involving sex of face judged (all $\mathrm{F}<3.0$, all

$405 \mathrm{p}>.08$, all partial eta $\left.{ }^{2}<.03\right)$.

406

INSERT FIGURE 3 AROUND HERE

408

409

Discussion

410 The proportion of trials on which women judged masculinized versions of

411 faces to be more dominant than feminized versions was increased after

412 viewing a slideshow of images of angry men, but not after viewing slideshows

413 of angry women or smiling faces of either sex. Since previous work has

414 shown that viewing images of angry men increases self-protection motivations

415 (Ackerman et al., 2006; Becker et al., 2007), these findings support the

416 proposal that dominance perceptions simply function to identify individuals

417 who are capable of inflicting substantial physical harm so that the perceiver

418 can respond to them in ways that maximize their own physical safety

419 (Oosterhof \& Todorov, 2008), at least in women.

421 By contrast with our findings for women's dominance perceptions, men's

422 dominance perceptions were modulated by the sex of the faces they were

423 exposed to during the priming phase, regardless of the emotional expression

424 those faces displayed; the proportion of trials on which men chose 
425

426

427

428

429

430

431

432

433

434

435

masculinized faces as more dominant tended to be increased after viewing images of women's faces, but tended to be decreased after viewing images of men's faces. Thus, although the changes in perception between the prepriming and post-priming tests were not significant in either the male or female priming sex conditions $(p=.10$ and $p=.13$, respectively), these changes were significantly different from one another, demonstrating that priming sex had the predicted effect on men's dominance perceptions. More female biased-sex ratios are associated with increased direct (i.e., violent) competition for resources (Barber, 2011; Del Giudice, 2012), potentially because female biased-sex ratios are correlated with lower relationship commitment and greater sexual promiscuity (Barber, 2000, 2009, 2011; Schmitt, 2005). Furthermore, viewing female-biased or male-biased slideshows recalibrates behaviors and perceptions in ways that suggest recent visual experience recalibrates impressions of the sex-ratio of the local population (Griskevicius et al., 2012; Watkins et al., 2012a). Thus, the observed effect of priming sex on men's dominance perceptions supports the proposal that dominance perceptions in men are relatively specialized and function primarily to facilitate effective direct intrasexual competition for resources (Puts, 2010).

Griskevicius et al. (2012) recently reported that priming men with cues to a male-biased local population increased the extent to which they sacrificed long-term financial gains for smaller, immediate financial gains. Importantly, our results, which suggest that priming men with cues to a female-biased local population triggers changes in men's dominance perceptions that might 
450 function to support effective violent competition for mates, are not

451 incompatible with Griskevicius et al's (2012) findings; while our results appear

452 to tap behaviors relating to direct (i.e., violent) competition for mates,

453 Griskevicius et al's (2012) results appear to tap behaviors relating to more

454 indirect, non-violent competition for economic resources. Indeed, when

455 considered together, the differences between our and Griskevicius et al's

456 (2012) findings complement the differences among correlational studies in

457 which female-biased sex ratios were found to be positively correlated with

458 violent crime rates, while male-biased sex ratios were found to be positively

459 correlated with the intensity of indirect (i.e., non-violent) competition for

460 access to financial resources among men (Barber, 2000, 2009, 2011).

461

462 That the effect of priming sex on men's dominance perceptions was not

463 qualified by a higher order interaction involving priming emotion suggests that

464 the priming effect observed for men in our experiment is not simply due to

465 viewing images of women priming men's sexual motivation. Although previous

466 studies have suggested that priming men's sexual motivation with images of

467 women can influence their behavioral responses, these effects occur only

468 when men are primed with images of attractive women and do not occur when

469 men are primed with images of relatively unattractive women (e.g., Wilson \&

470 Daly, 2004). Since smiling has previously been shown to increase women's

471 attractiveness and to elicit approach responses from men in courtship

472 contexts (reviewed in Gueguen, 2008), the absence of an interaction between

473 the effects of priming sex and priming emotion on men's dominance

474 perceptions is difficult to explain in terms of increased sexual motivation. 
476 Given the proposal that men's dominance perceptions may be somewhat specialized to facilitate effective direct (i.e., violent) intrasexual competition for mates (Puts, 2010), one might have expected the effect of cues to the sex ratio of the local population on men's dominance perceptions to occur for 480 judgments of men's, but not women's, dominance. Similarly, if women's 481 dominance perceptions are closely related to self-protection motivations, one 482 might have expected the priming effect for female participants to be greater 483 for judgments of men's than women's faces, given sex differences in physical 484 strength and aggression (Archer, 2009; Sell et al., 2009). Although our data 485 show that masculinization had a greater overall effect on judgments of men's 486 dominance than on judgments of women's dominance (see also Watkins et 487 al., 2010a), suggesting that physical dominance cues may generally be more 488 salient in men's than women's faces, neither the effect of priming sex that was 489 observed for male participants nor the interaction between priming sex and 490 priming emotion that was observed for female participants were qualified by 491 higher order interactions involving the sex of the faces judged in the 492 dominance perception tests. These patterns of results may have occurred 493 because changes in perceptions of women's dominance are a relatively low494 cost, functionless byproduct of perceptual processes that evolved primarily to 495 recalibrate perceptions of men's dominance in light of current environmental 496 factors (i.e., there is little cost to changing dominance perceptions generally, 497 rather than altering them for men's faces only). Alternatively, it is possible that 498 the role of women's physical dominance in perceptions and behaviors related 499 to both violent conflict and resource holding has been underestimated in 
500 previous work. Consistent with the former proposal, facultative preferences for

501 sexually dimorphic facial cues have been shown to occur for both own-sex

502 and opposite-sex faces in circumstances where the change in perceptions of

503 own-sex faces served no obvious function (e.g., Welling et al., 2007).

504 Consistent with the latter proposal, however, Sell et al. (2009) have shown

505 that participants can assess the physical strength and fighting ability of

506 women from facial photographs somewhat accurately (albeit less accurately

507 than they can make the corresponding judgments for male faces),

508 demonstrating the existence of psychological adaptations for assessing

509 women's physical dominance. Our current data do not distinguish between

510 these two possibilities.

511

512 Although we used somewhat indirect methods for manipulating motivations

513 relevant to self-protection and within-sex competition for mates, it is worth

514 noting here that there is considerable evidence for the validity of these

515 techniques. For example, previous studies have presented evidence that 516 exposure to angry faces, and angry men in particular, triggers perceptual

517 responses that might function to decrease risk of physical injury, particularly

518 among those individuals who are least able to defend themselves physically

519 (reviewed in Kenrick et al., 2010). A similar pattern of results is also evident

520 in our own data, in which women showed increased dominance sensitivity

521 after viewing images of angry men. There is also now evidence that

522 experimentally manipulating cues to the sex ratio of the local population

523 during priming phases of experiments triggers behaviors that are similar to

524 those seen in correlational studies in which naturally occurring variation in sex 
525 ratios predicted (i.e., was correlated with) variation in human behavior;

526 experiments show that increasing cues that mates are abundant in the local

527 population causes men to value financial resources more (Griskevicius et al.,

528 2012) and women to become choosier in their mate preferences (Watkins et

529 al., 2012). These patterns of results have also been observed in correlational

530 studies in which naturally occurring variation in sex ratios was correlated with

531 the extent to which men compete for financial resources (Barber, 2009; Del

532 Giudice, 2012) and measures of women's choosiness in their mate choices

533 (Pollet \& Nettle, 2008). That the current study found that increasing cues that

534 competitors for mates are abundant in the local population causes men to be

535 more sensitive to cues of other men's dominance continues this theme of

536 priming experiments and correlational studies showing similar patterns of

537 results; correlational studies suggest that indices of violent competition for

538 mates among men are greater in regions with more female-biased sex ratios

539 (Barber, 2011; Del Giudice, 2012). Collectively, these results suggest that

540 interpretations of our findings for women's and men's dominance perceptions

541 that emphasize self-protection motivations and within-sex competition for

542 mates, respectively, are justified. Indeed, while correlational studies suggest

543 that sex ratio predicts non-violent competition for resources and violent

544 competition for mates among men in different ways, our findings, together

545 with those reported by Griskevicius et al. (2012) suggest that experimentally

546 manipulating cues to the sex ratio of the local population may also have

547 different effects on these two different types of competition among men.

548 Exploring this possibility further may be a fruitful line of research. 
550 We suggest that our findings are best explained by sex-specific responses to

551 cues to probable conditions in the local population. However, recent visual

552 experience with faces can also influence social judgments via perceptual

553 aftereffects, whereby viewing faces that possess a specific characteristic

554 decreases sensitivity to that characteristic in previously unseen faces

555 (reviewed in Webster et al., 2011). However, we suggest that our findings are

556 unlikely to reflect this type of perceptual aftereffect for three reasons. First,

557 aftereffects induced by exposure to faces of a given sex or displaying a given

558 emotional expression are typically equivalent in men and women (Webster et

559 al., 2011). By contrast, our results for recent visual experience and dominance

560 perceptions were different for male and female participants. Second,

561 perceptual aftereffects do not generally transfer well from one sex of face to

562 the other (e.g., Little et al., 2005) and, if they do, the size of the aftereffects is

563 generally significantly smaller than when the faces shown in the exposure and

564 test phases were the same sex (e.g., Jacquet \& Rhodes, 2008). By contrast

565 with this typical pattern for face aftereffects, the effect of viewing male or

566 female faces on men's dominance perceptions in our experiment was

567 unaffected by the sex of the faces judged in the test phases. Third, emotion

568 aftereffects induced by viewing male or female faces are typically similar in

569 magnitude (e.g., Bestelmeyer et al., 2010). By contrast, our findings for

570 women's dominance perceptions suggest that viewing angry facial

571 expressions in the context of male and female faces cause very different

572 patterns of results. Together, these lines of reasoning mean that it is very

573 difficult to explain our findings in terms of perceptual aftereffects alone.

574 Nonetheless, we acknowledge that converging evidence for sex-specific 
575 context-sensitivity in dominance judgments from studies using other types of

576 priming techniques may well be needed to clarify the interpretation of our

577 findings.

578

579 Most previous work on facultative responses to facial cues has investigated

580 the effects of environmental factors on judgments of others' attractiveness

581 (reviewed in, e.g., Little et al., 2011). By contrast with this emphasis on mate

582 preferences, our findings add to a growing literature suggesting the existence

583 of facultative perceptions of others' dominance (e.g., Burriss \& Little, 2006;

584 Watkins \& Jones, 2012). However, while these previous studies focused on

585 men's judgments of other men's dominance, here we show that women's

586 perceptions of others' dominance can also be influenced by contextual

587 factors. The facultative nature of dominance perceptions, and social

588 judgments in general, may be important given that they tie up cognitive and

589 perceptual resources, which are finite and should be allocated judiciously

590 (Kenrick et al., 2010). Thus, modulating social judgments, such as dominance

591 perceptions, according to the demands of one's own current circumstances

592 (e.g., in light of cues that one's own safety may be at risk or that direct

593 competition for resources is likely to be particularly intense) may help

594 individuals to allocate their cognitive and perceptual resources efficiently.

595 Additionally, heightened sensitivity to dominance cues in situations where

596 violence is uncommon and there is little direct competition for resources may

597 be counterproductive if it, for example, reduces the pool of potential co-

598 operators and allies. 
600 Here we show that the salience of facial cues of physical dominance is

601 increased when women are primed with images of angry men, but not images

602 of angry women or smiling individuals of either sex. This result suggests that

603 activating self-protection motivations increases the salience of cues of others'

604 dominance, supporting the proposal that dominance perceptions primarily

605 function to identify individuals who are able to inflict physical harm so that the 606 perceiver can respond in ways that maximize their own safety (Oosterhoff \&

607 Todorov, 2008), in women at least. We also show that the salience of facial 608 cues of physical dominance is greater when men are primed with images of

609 women than when they are primed with images of men, regardless of the

610 emotional expressions displayed on these priming images. This result

611 suggests that cues to the sex ratio of the local population biases men's

612 dominance perceptions, supporting the proposal that dominance perceptions

613 in men are relatively specialized for effective direct intrasexual competition for

614 resources (Puts, 2010). Together, these sex-specific priming effects provide

615 new insights into the routes through which physical violence and intrasexual

616 competition for resources may have shaped the visuo-cognitive processes

617 that support social interactions by revealing a sex difference in the effects of

618 cues to the local environment on perceptions of others' dominance. While

619 men's dominance perceptions appear to be primarily sensitive to factors

620 relating to direct intrasexual competition, women's dominance perceptions

621 appear to function primarily to protect themselves from physical harm more

622 generally.

623

624 References 
625 Ackerman, J., Shapiro, J. R., Neuberg, S. L., Kenrick, D. T., Schaller, M., Becker, D. V. et al. (2006). They all look the same to me (unless they're angry): From out-group homogeneity to out-group heterogeneity. Psychological Science, 17, 836-840.

Archer, J. (2009). Does sexual selection explain human sex differences in aggression? Behavioral and Brain Sciences, 32, 249-266.

Barber, N. (2011). Marriage markets and mating aggression help explain societal differences in violent crime. Aggression and Violent Behavior, 16, 420-427.

Barber, N. (2009). Countries with fewer males have more violent crime: Marriage markets and mating aggression. Aggressive Behavior, 35, 4956.

Barber, N. (2000). The sex ratio as a predictor of cross-national variation in violent crime. Cross Cultural Research, 34, 264-282. Journal of Personality and Social Psychology, 92, 179-190.

642 Bestelmeyer, P. E., Jones, B. C., DeBruine, L. M., Little, A. C. \& Welling, L. L. M. (2010). Face aftereffects demonstrate interdependent processing of

646 Burriss, R. P. \& Little, A. C. (2006). Effects of partner conception risk phase on male perception of dominance in faces. Evolution and Human Behavior, 27, 297-305. 
649 DeBruine, L. M., Jones, B. C., Smith, F. G. \& Little, A. C. (2010). Are attractive 650 men's faces masculine or feminine? The importance of controlling confounds in face stimuli. Journal of Experimental Psychology: Human Perception and Performance, 36, 751-758.

653 DeBruine, L. M., Jones, B. C., Little, A. C., Boothroyd, L. G., Perrett, D. I., Penton-Voak, I. S., Cooper, P. A., Penke, L., Feinberg, D. R. \& Tiddeman, B. P. (2006). Correlated preferences for facial masculinity and ideal or actual partner's masculinity. Proceedings of the Royal Society of London B, 273, 1355-1360.

Del Giudice, M. (2012). Sex ratio dynamics and fluctuating selection on personality. Journal of Theoretical Biology, 297, 48-60.

660 Fink, B., Neave, N. \& Seydel, H. (2007). Male facial appearance signals physical strength to women. American Journal of Human Biology, 19, 82-87.

663 Fox, E., Russo, R., Bowles, R. \& Dutton, K. (2001). Do threatening stimuli draw or hold visual attention in subclinical anxiety? Journal of Experimental Psychology: General, 130, 681-700.

666 Griskevicius, V., Tybur, J. M., Ackerman, J. M., Delton, A. W. \& Robertson, T. E. (2012). The financial consequences of too many men: Sex ratio effects on saving, borrowing and spending. Journal of Personality and

670 Gueguen, N. (2008). The effect of a woman's smile on men's courtship behavior. Social Behavior \& Personality, 36, 1233-1236. 
672 Jacquet, E. \& Rhodes, G. (2008). Face aftereffects indicate dissociable, but not distinct, coding of male and female faces. Journal of Experimental Psychology Human Perception and Performance, 34, 101-112.

675 Jones, B. C., DeBruine, L. M., Main, J. C., Little, A. C., Welling, L. L. M., Feinberg, D. R. \& Tiddeman, B. P. (2010). Facial cues of dominance modulate the short-term gaze-cuing effect in human observers. Proceedings of the Royal Society of London B, 277, 617-624.

Jones, B. C., DeBruine, L. M., Little, A. C., Burriss, R. P. \& Feinberg, D. R. (2007). Social transmission of face preferences among humans. Proceedings of the Royal Society of London B, 274, 899-903.

Keating, C. F. \& Bai, D. L. (1986). Children's attributions of social dominance 683 from facial cues. Child Development, 57, 1269-1276.

Keating, C. F., Mazur, A. \& Segall, M. H. (1981). A cross-cultural exploration of physiognomic traits of dominance and happiness. Ethology and Sociobiology, 2, 41-48.

Kenrick, D. T., Neuberg, S. L., Griskevicius, V., Becker, D. V. \& Schaller, M. 688 (2010). Goal-driven cognition and functional behavior: The fundamental-motives framework. Current Directions in Psychological Science, 19, 63-67.

691 Little, A. C., Jones, B. C. \& DeBruine, L. M. (2011). Facial attractiveness: Evolutionary based research. Philosophical Transactions of the Royal Society $B, 366,1638-1659$.

694 Little, A. C., DeBruine, L. M., \& Jones, B. C. (2005). Sex-contingent face aftereffects suggest distinct neural populations code male and female faces. Proceedings of the Royal Society of London B, 272, 2283-2287. 
Lundqvist, D. \& Litton, J. E. (1998). The averaged Karolinska directed emotional faces-AKDEF. AKDEF CDROM Stockholm, USA: Psychology section, Karolinska Institute.

Oosterhof, N. N., \& Todorov, A. (2008). The functional basis of face evaluation. Proceedings of the National Academy of Sciences, 105, 11087-11092.

Perrett, D. I., Lee, K. J., Penton-Voak, I. S., Rowland, D. R., Yoshikawa, S., Burt, D. M., Henzi, S. P., Castles, D. I. \& Akamatsu, S. (1998). Effects of sexual dimorphism on facial attractiveness. Nature, 394, 884-887.

Pollet, T. V. \& Nettle, D. 2008. Driving a hard bargain: Sex ratio and male marriage success in a historical US population. Biology Letters, 4, 3133.

Puts, D. A., Apicella, C. L. \& Cardenas, R. A. (2011). Masculine voices signal men's threat potential in foraging and industrial societies. Proceedings of the Royal Society of London B, DOI: 10.1098/rspb.2011.0829.

Puts, D.A. (2010). Beauty and the beast: Mechanisms of sexual selection in humans. Evolution and Human Behavior, 31, 157-175.

Schmitt, D. P. (2005). Sociosexuality from Argentina to Zimbabwe. A 48nation study of sex, culture, and strategies of human mating. Behavioral and Brain Sciences, 28, 247-311.

Scott, I., Swami, V., Josephson, S. C. \& Penton-Voak, I. S. (2008). Contextdependent preferences for facial dimorphism in a rural Malaysian population. Evolution and Human Behavior, 29, 289-296.

Sell, A., Cosmides, L., Tooby, J., Sznycer, D., von Rueden, C. \& Gurven, M. (2009). Human adaptations for the visual assessment of strength and 
fighting ability from the body and face. Proceedings of the Royal Society of London B, 276, 575-584.

Senior, C., Barnes, J., Jenkins, R., Landau, S., Philips, M. L., \& David, A. S. (1999a). Attribution of social dominance and maleness to schematic faces. Social Behavior and Personality, 27, 331-338.

Senior, C., Philips, M. L., Barnes, J., \& David, A. S. (1999b). An investigation into the perception of dominance from schematic faces: A study using the World-Wide Web. Behavior Research Methods, Instruments, \& Computers, 31, 341-346.

Stevens, J. P. (2007). Intermediate Statistics: A Modern Approach. Lawrence Earlbaum, NY.

Tiddeman, B. P., Burt, D. M., \& Perrett, D. I. (2001). Prototyping and transforming facial texture for perception research. IEEE Computer Graphics and Applications, 21, 42-50.

Undurraga, E. A., Eisenberg, D. T. A., Oyunbileg, M., Wang, R., Leonard, W. R., McDade, T. W., Reyes-Garcia, V., Nyberg, C., Tanner, S., Huanca, T., TAPS. Bolivia Study Team \& Godoy, R. A. (2010). Human's cognitive ability to assess facial cues from photographs: A study of sexual selection in the Bolivian Amazon. PLoS ONE, 5, e11027.

Watkins, C. D. \& Jones, B. C. (2012). Priming men with different contest outcomes modulates their dominance perceptions. Behavioral Ecology, 23, 539-543.

Watkins, C. D., Jones, B. C., Little, A. C., DeBruine, L. M. \& Feinberg, D. R. (2012a). Cues to the sex ratio of the local population influence 
women's preferences for facial symmetry. Animal Behaviour, 83, 545553.

Watkins, C. D., Quist, M., Smith, F. G., DeBruine, L. M. \& Jones, B. C. (2012b). Individual differences in women's perceptions of other women's dominance. European Journal of Personality, 26, 79-86.

751
B. C. \& DeBruine, L. M. (2010a). Individual differences in dominance perception: Dominant men are less sensitive to facial cues of male dominance. Personality and Individual Differences, 49, $967-971$.

Watkins, C. D., Fraccaro, P. J., Smith, F. G., Vukovic, J., Feinberg, D. R., DeBruine, L. M. \& Jones, B. C. (2010b). Taller men are less sensitive to cues of dominance in other men. Behavioral Ecology, 21, 943-947.

Webster, M. A. \& MacLeod, D. I. A. (2011). Visual adaptation and face perception. Philosophical Transactions of the Royal Society B, 366, $1702-1725$.

Welling, L. L. M., Jones, B. C., DeBruine, L. M., Smith, F. G., Feinberg, D. R., Little, A. C. \& Al-Dujaili, E. A. S. (2008). Men report stronger attraction to femininity in women's faces when their testosterone levels are high. Hormones and Behavior, 54, 703-708.

Welling, L. L. M., Jones, B. C., DeBruine, L. M., Conway, C. A., Law Smith, M. J., Little, A. C., Feinberg, D. R., Sharp, M. \& Al-Dujaili, E. A. S. (2007). Raised salivary testosterone in women is associated with increased attraction to masculine faces. Hormones and Behaviour, 52, 156-161. 
769 Wilson, M. \& Daly, M. (2004). Do pretty women inspire men to discount the

770

771

772

773

774

775

776 future? Proceedings of the Royal Society of London B, 271, S177S179.

Windhager, S., Schaefer, K. \& Fink, B. (2011). Geometric morphometrics of male facial shape in relation to physical strength and perceived attractiveness, dominance, and masculinity. American Journal of Human Biology, 23, 805-814. 
777 Table 1. Mean proportion of trials (+/- SEM) for each combination of

778 participant sex (male, female), sex of face judged (male, female), test phase

779 (pre-priming, post-priming), priming emotion (angry, smiling), and priming sex

780 (male, female).

781

\begin{tabular}{|c|c|c|c|c|c|}
\hline $\begin{array}{c}\text { participant } \\
\text { sex }\end{array}$ & priming & $\begin{array}{c}\text { pre-priming } \\
\text { and male } \\
\text { faces }\end{array}$ & $\begin{array}{c}\text { pre-priming } \\
\text { and female } \\
\text { faces }\end{array}$ & $\begin{array}{c}\text { post- } \\
\text { priming and } \\
\text { male faces }\end{array}$ & $\begin{array}{c}\text { post- } \\
\text { priming and } \\
\text { female faces }\end{array}$ \\
\hline male & angry men & $.86(.03)$ & $.58(.06)$ & $.79(.04)$ & $.56(.07)$ \\
\hline male & angry women & $.87(.04)$ & $.52(.07)$ & $.85(.04)$ & $.60(.07)$ \\
\hline male & smiling men & $.87(.03)$ & $.56(.07)$ & $.80(.04)$ & $.57(.07)$ \\
\hline male & smiling & $.86(.03)$ & $.63(.06)$ & $.88(.03)$ & $.62(.07)$ \\
\hline female & women & & & & $.54(.02)$ \\
\hline female & angry men & $.88(.03)$ & $.55(.07)$ & $.59(.08)$ \\
\hline female & smiling men & $.87(.05)$ & $.58(.07)$ & $.88(.04)$ & $.56(.08)$ \\
\hline female & smiling & $.84(.05)$ & $.55(.07)$ & $.84(.05)$ & $.58(.08)$ \\
& women & & & & \\
\hline
\end{tabular}

782

783

784 
Figure 1.
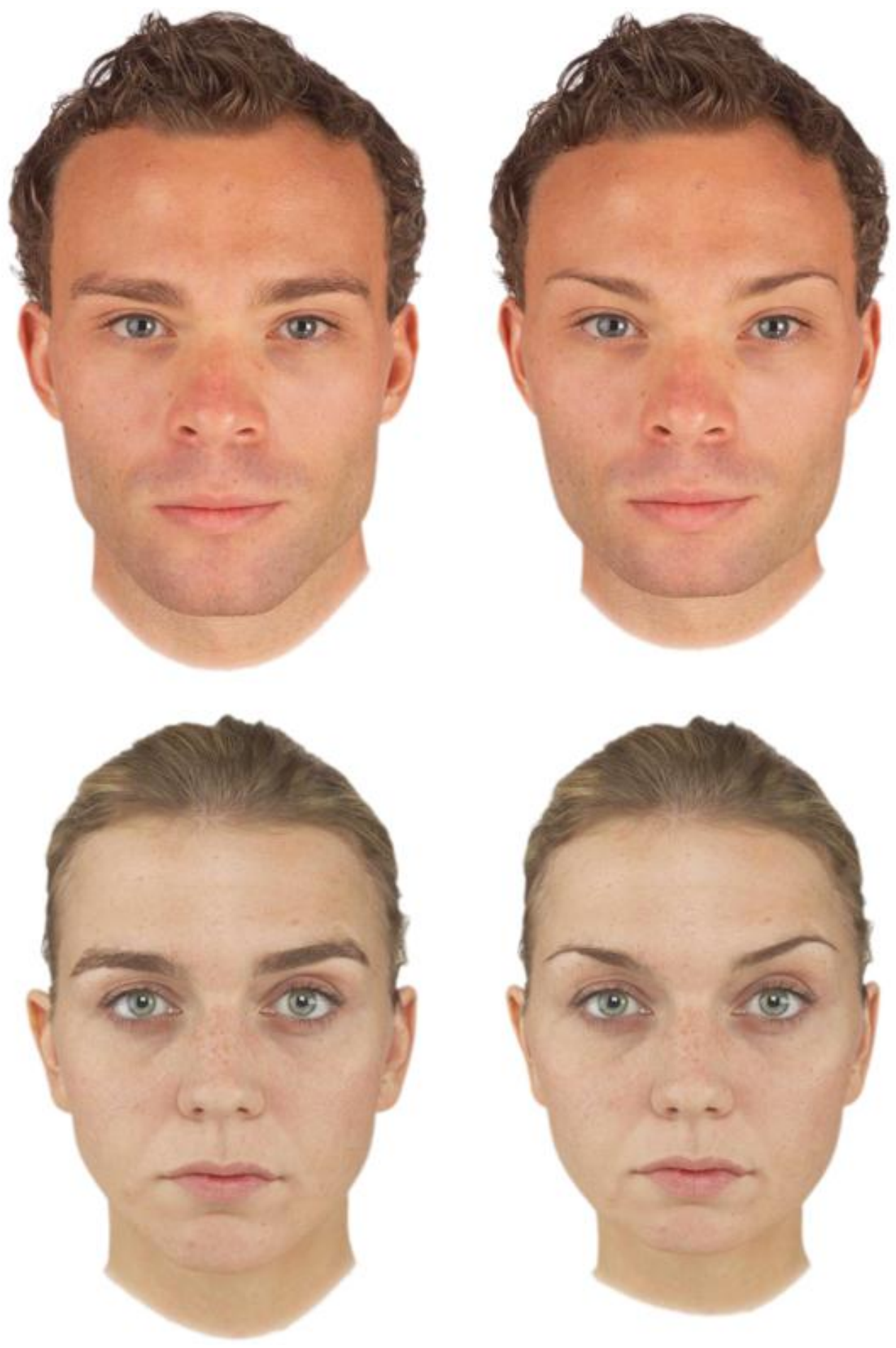

788 Figure 1. Examples of face stimuli used to assess dominance perceptions.

789 Masculinized versions of face images are shown in the left column and

790 feminized versions in the right column. 
Figure 2.

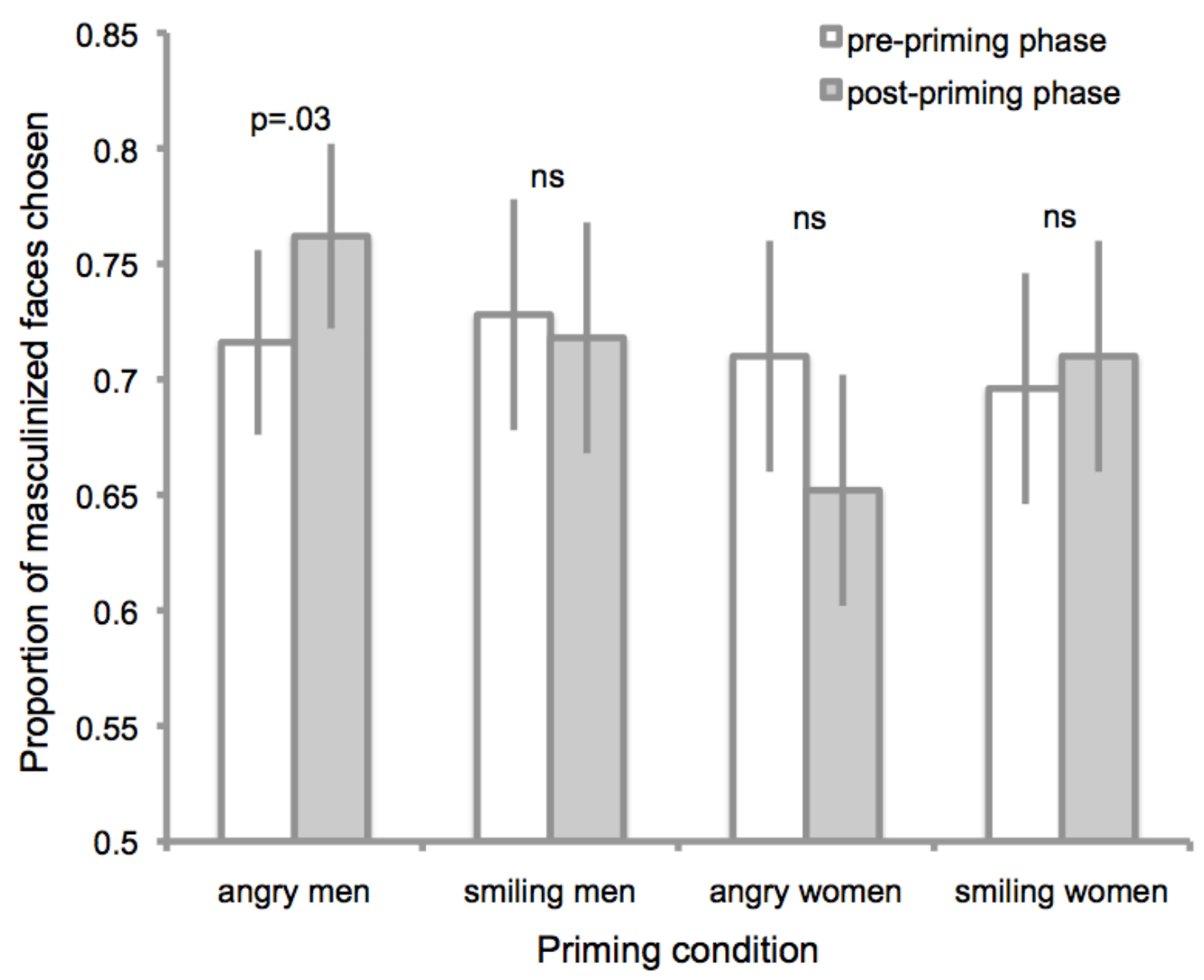

795 Figure 2. The significant three-way interaction among test phase, priming 796 emotion, and priming sex that was observed for female participants. Women 797 who were primed with angry male images, but not women primed with angry 798 female images or smiling images of either sex, significantly increased the 799 proportion of masculinized faces chosen as more dominant between the pre800 priming and post-priming tests ( $p$ values indicate the results of paired samples 801 t-tests). 
803

804

\section{Figure 3.}

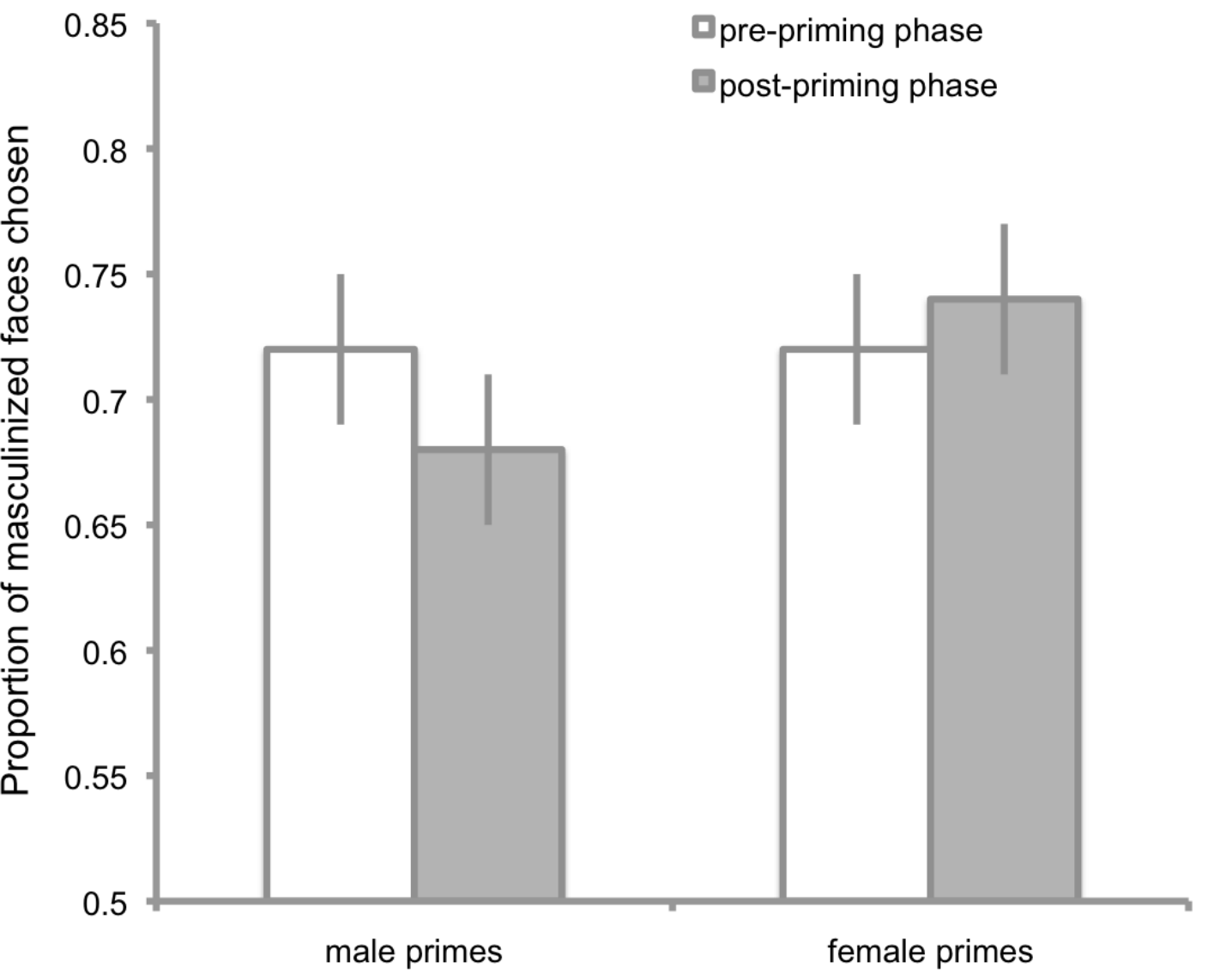

806

807 Figure 3. The significant two-way interaction between test phase and priming 808 sex that was observed for male participants. Men who were primed with male 809 images tended to decrease the proportion of masculinized faces they chose

810 as more dominant between the pre-priming and post-priming phases $(p=.10)$

811 and men who were primed with female images tended to increase the 812 proportion of masculinized faces they chose as more dominant between the 813 pre-priming and post-priming phases $(p=.13)$.

814 